\title{
The cellular repressor of E1A-stimulated genes mediates glucocorticoid-induced loss of the type-2 IGF receptor in ileal epithelial cells
}

\author{
P V Gordon, J B Paxton and N S Fox ${ }^{1}$ \\ Department of Pediatrics, Division of Neonatology, University of Virginia Health Sciences, PO Box 800386, Charlottesville, Virginia 22908, USA \\ ${ }^{1}$ Department of Microbiology, University of Virginia Health Sciences, PO Box 800734, Charlottesville, Virginia 22908, USA \\ (Requests for offprints should be addressed to P V Gordon; Email: pvg4n@virginia.edu)
}

\begin{abstract}
Glucocorticoids induce hypertrophy of the neonatal ileal mucosa but the molecular mechanisms behind this growth induction remain poorly understood. Ileal epithelial cells (IECs) are dependent upon IGF-II for proliferation both in vivo and in culture. The type-2 IGF receptor (IGFR-2) is a lysosomal transport protein that attenuates IGF-IIdriven growth and is highly abundant in the ileum. The cellular repressor of E1A-stimulated genes (CREG) is a secreted phosphoglycoprotein that affects cell fate via ligand binding with IGFR-2, although the mechanism by which it does so is unknown. We hypothesized that glucocorticoids might facilitate IGF-mediated hypertrophy through CREG-mediated degradation of IGFR-2. To test this hypothesis, confluent rat IECs (IEC-18) were cultured for $72 \mathrm{~h}$ with or without dexamethasone (DEX) and harvested for Western blot, immunocytochemistry, gene array and CREG immunoneutralization experiments.
\end{abstract}

IGFR-2 and CREG immunohistochemistry were also performed in archived ileal specimens from control and DEX-exposed newborn mice and extremely premature infants to investigate in vivo and clinical relevance. DEX exposure was found to diminish IGFR-2 immunolocalization in cultured rat IECs, newborn mouse ileal mucosa and human neonatal ileal mucosa. Gene array data indicated that IGFR-2 expression was unchanged with DEX treatment, suggesting a mechanism of protein degradation. CREG immunolocalization and abundance was found to be increased by DEX and immunoneutralization of CREG resulted in the abolition of IGFR-2 degradation. We have concluded that CREG is a secreted mediator by which DEX induces degradation of IGFR-2 and speculate that this is a fundamental mechanism of mucosal growth induction.

Journal of Endocrinology (2005) 185, 265-273

\section{Introduction}

Glucocorticoids trigger mucosal maturation during weaning and can accelerate the process if given exogenously in the week immediately prior to weaning (Henning \& Sims 1979, Oesterreicher et al. 1998, Solomon et al. 2001). However, there is a limited temporal window in which this endocrine stimulus is optimal (Solomon et al. 2001). In contrast, we have previously reported a significant increase in ileal epithelial and goblet cell numbers when newborn mice are treated with early postnatal dexamethasone (DEX) (Gordon et al. 2001a), suggesting a proliferative mechanism of hypertrophy that is potentially ever present in the neonate. Similar findings have been reported in fetal sheep when exposed to antenatal steroids, indicating that the capacity extends backwards into gestation (Trahair et al. 1987). These paradigms have clinical relevance to an emerging and highly morbid neonatal disease known as spontaneous intestinal perforation (also called focal small bowel perforation) which afflicts $5-10 \%$ of all extremely low birth weight infants (Garland et al. 1999, Gordon et al. 1999, 2001b, Stark et al. 2001). At present, the precise mechanisms by which glucocorticoids induce perforations is unknown but many growth factor perturbations have now been demonstrated with DEX administration in the newborn mouse ileum (Gordon et al. 2001a,c,d, 2002, 2004, Herman et al. 2004). These growth factor disturbances are associated with structural skewing of the neonatal ileum via two opposing trophisms: (1) mucosal hypertrophy (which is associated with distention of the distal bowel) and (2) bowel wall atrophy (which is associated with thinning of the submucosa and focal necrosis of the muscularis) (Gordon et al. 2002, Herman et al. 2004).

Fetal gut epithelial cell proliferation is dependent upon insulin-like growth factor-II (IGF-II) (Tremblay et al. 2001). The type-2 IGF receptor (IGFR-2) is a lysosomal transport protein that captures free IGF-II and traffics it to 
the pre-lysosomal compartment, thereby targeting it for degradation. The net function of this activity is to attenuate IGF-II-driven proliferation and growth. During gestation, IGFR-2 serves a crucial global function because it prevents fetal overgrowth and birth-related injury due to fetal-pelvic disproportion (Lau et al. 1994, Wang et al. 1994, D'Ercole 1999, Killian et al. 2000, 2001a, Young et al. 2001). However, release from IGFR-2 growth attenuation in the perinatal period is a developmental event that is not well studied and could be sensitive to glucocorticoids.

The IGFR-2 protein is encoded by an imprinted gene that diverged relatively recently (coincident with marsupialization and the evolution of the placenta) from the mannose-6-phosphate transporter - a very old gene which can be traced phylogenetically all the way back to yeast (Morgan et al. 1987, MacDonald et al. 1988, Clairmont \& Czech 1989, Dahms et al. 1993, Yandell et al. 1999, Killian et al. 2001b, Whyte \& Munro 2001). Despite this wealth of knowledge, we know very little about how IGFR-2 is regulated. One candidate regulatory agent that is known to bind to IGFR-2 is the cellular repressor of E1A-stimulated genes (CREG) (Clairmont \& Czech 1989, Veal et al. 1998, 2000). This secreted $28 \mathrm{kDa}$ phospho-glycoprotein is known to require the presence of IGFR-2 in order to alter cell fate in teratoma cultures (Veal et al. 2000). While the mechanism by which CREG causes transformation is unknown, one hypothesis is that CREG binds IGFR-2 and drives it into the lysosome resulting in increased IGF-II signaling (illustrated in Fig. 1). This condition could be permissive for cell fate change (and quiescence) in some cell types, but could facilitate proliferation in others. This would be an alternate method of degradation from the norm, which consists of cell surface proteolysis (DiBacco \& Gill 2003). If this hypothesis is true, CREG might mediate glucocorticoid-induced degradation of IGFR-2 in ileal epithelial cells (IECs) and facilitate proliferation.

\section{Materials and Methods}

\section{Cell culture}

Rat ileal epithelial crypt cells (IEC-18; American Type Culture Collection, Rockville, MD, USA) from aliquots of passage numbers 6-8 were grown to confluence in Dulbecco's modified Eagle's medium (DMEM) with 10\% fetal bovine serum and $0 \cdot 01 \%$ insulin in six-well plates or $150 \mathrm{~mm}$ dishes. The time-period for complete epithelial cell confluence was 24-48 h. Confluent cells were incubated for $72 \mathrm{~h}$ in DMEM with $10^{-6} \mathrm{M}$ DEX (Sigma), $10^{-6}$ M NBI 31772 (Calbiochem, San Diego, CA, USA) or no treatment as control. After $72 \mathrm{~h}$, the media were aspirated and the cells were harvested for either immunocytochemistry or Western blot analysis.

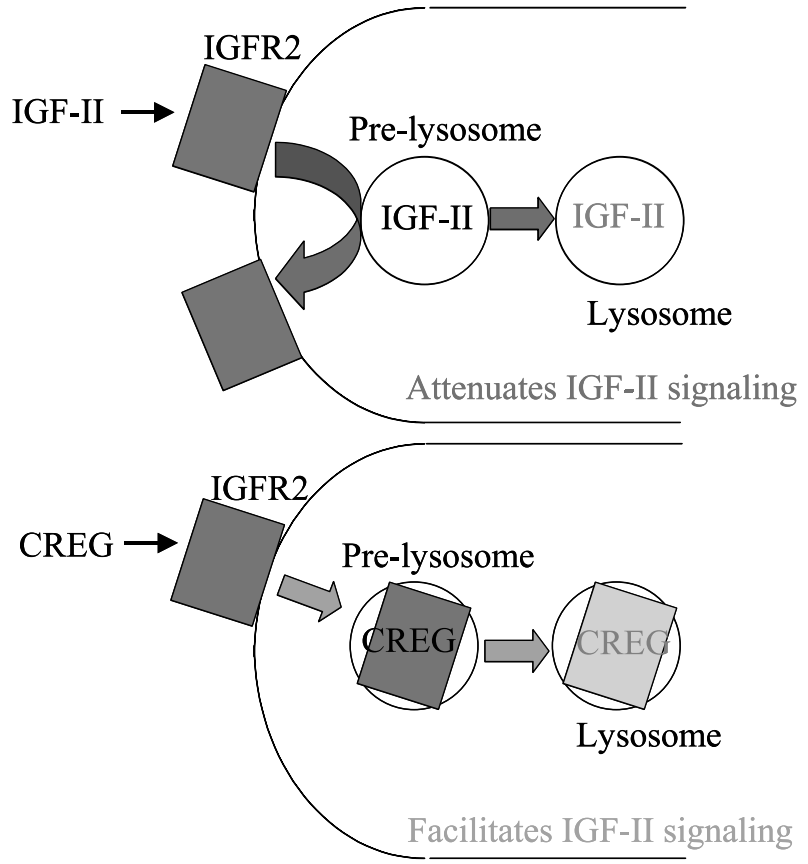

Figure 1 Illustration of our hypothesized mechanism by which CREG degrades IGFR-2. In the top half, IGF-II is shown binding to IGFR-2 on the cell surface where it is internalized, trafficked to the pre-lysosome and released - allowing IGFR-2 to be recycled back to the cell surface. IGF-II is then degraded in the lysosome and the net effect is to attenuate IGF-II signaling for that cell. In the bottom half, CREG binds to IGFR-2, causes it to internalize, prevents uncoupling and then drives it into the lysosome thereby causing degradation of both. The net effect is to facilitate IGF-II signaling by decreasing IGFR-2 abundance.

\section{Immunocytochemistry}

Immunocytochemistry was performed for IGFR-2 and CREG in IEC-18 cells grown on glass coverslips in six-well plates, with separate treatment conditions batched and processed in parallel for each antibody. After being fixed in formalin overnight, the cells were washed in DIG buffer $(40 \mathrm{mM}$ Tris base, $300 \mathrm{mM}$ Tris $\mathrm{HCl}$ and $150 \mathrm{mM}$ $\mathrm{NaCl})$ five times, then put in blocking solution $(1 \%(\mathrm{w} / \mathrm{v})$ bovine serum albumin (BSA) in DIG buffer) for $1 \mathrm{~h}$ at room temperature (RT). We used a 1-h incubation of primary antibody (all were obtained from Santa Cruz Biotechnology, Santa Cruz, CA, USA) at RT for all studies. Primary antibody was washed off with DIG $\times 5$ and biotin-conjugated secondary antibody (all obtained from Jackson Immunoresearch Laboratories, West Grove, PA, USA) was placed on the slides for $1 \mathrm{~h}$ and then washed again in the same fashion. Signal amplification and detection was performed using standard $\mathrm{ABC}$ biotinconjugated horseradish peroxidase (ABC-HRP; according to the manufacturer's recommendation; Elite Series, Vector Laboratories, Burlingame, CA, USA). The slides were counterstained with hematoxylin and cover-slipped for microscopy. 


\section{Western blots}

Cells were collected from $150 \mathrm{~mm}$ dishes, washed with phosphate-buffered serum and recovered in $700 \mu \mathrm{l}$ lysis buffer $(50 \mathrm{mM}$ Tris- $\mathrm{HCl}, 150 \mathrm{mM} \mathrm{NaCl}$ and 1\% NP$40 \%$ in RNase-free water) by scraping the dish. Cells were spun down for $10 \mathrm{~min}$ in a pre-chilled centrifuge. The supernatant was diluted 1:4 with sample buffer $(62.5 \mathrm{mM}$ Tris-HCl, $\mathrm{pH}$ 6.8, 20\% glycerol, 2\% SDS and 5\% $\beta$-mercaptoethanol) and run on $12 \%$ acrylamide gels. After the gel had been transferred onto a nitrocellulose membrane, $0.5 \%$ Ponceau S stain was applied to confirm the standardized protein concentrations.

Western blot membranes were blocked in 5\% freezedried non-fat cow's milk block dissolved in Tris-buffered saline (TBS)-Tween for $1 \mathrm{~h}$ at RT. Goat polyclonal primary antibody for IGFR-2, CREG or IGF-binding protein (IGFBP)-3 as a loading comparison control (all antibodies obtained from Santa Cruz Biotechnology) were applied for $1 \mathrm{~h}$ at RT. Primary antibody was washed off with TBS-Tween, and biotin-conjugated secondary antibody (all from Jackson Immunoresearch Laboratories) was placed on the membrane for $1 \mathrm{~h}$ then washed again. ABC-HRP chemiluminescent visualization was performed according to the manufacturer's instructions.

\section{Gene array analysis}

IEC-18 cells were treated and processed as described in the section on Cell culture. Total RNA was harvested for each treatment condition in triplicate experiments and then used for gene chip analysis according to the manufacturer's protocol (Affymetrix, Santa Clara, CA, USA; rat gene chip no. 230 - a chip containing 9000 known genes - using the manufacturer's recommended internal controls and base-lining functions). Subset analyses were used to assess treatment effects between individual mRNA abundances within the IGF system and selected controls, these included: IGF-I, IGF-II, IGFR-1, IGFR-2, IGFBP-3, glucocorticoid-induced leucine zipper and glucocorticoidinduced protein kinase (with significant treatment differences defined as a $P$ value $<0.05$ in these subsets - using two-tailed paired t-test analysis - provided as part of the standard analysis by the University of Virginia Biomedical Research Facility and the Department of Health Evaluation Sciences).

\section{Tissue collection}

Our histological experiments utilized paraffin-blocked, formalin-fixed tissues retained from previously published studies (Gordon et al. 1999). The animal protocols in this study were reviewed and approved by the institutional animal care and use committee at the University of North Carolina. Newborn C57 wild-type mice were killed on day 3 of life (Gordon et al. 2001a). The ileum was removed, fixed in 10\% buffered formalin and embedded in paraffin. De-identified, paraffin-blocked, formalin-fixed neonatal ileums were obtained from age-matched autopsy controls that were not exposed to exogenous steroids, and surgical specimens from extremely low birth weight infants who received early postnatal DEX, acquired spontaneous intestinal perforation and then underwent immediate surgical resection (reviewed by the institutional review board at the University of Virginia). Eight control and eight DEX-treated newborn mice $($ dose $=1 \mu \mathrm{g} / \mathrm{g}$ per day for 3 days), and three autopsy controls and three DEX-treated neonates (maximum dose $=0.5 \mathrm{mg} / \mathrm{kg}$ per day over a 12-day taper) were examined.

\section{Immunohistochemistry}

Sections $(4 \mu \mathrm{m})$ were deparaffinized in xylene and hydrated in descending ethanol concentrations to distilled water. Antigen retrieval was performed by incubating slides in $0.1 \mathrm{M}$ citric acid, $\mathrm{pH} 8.0$, for $10 \mathrm{~min}$ at $100^{\circ} \mathrm{C}$, followed by cooling to $25^{\circ} \mathrm{C}$ and washing three times in distilled $\mathrm{H}_{2} \mathrm{O}$. Endogenous peroxidase activity was quenched in $3 \% \mathrm{H}_{2} \mathrm{O}_{2}$ in $60 \%$ methanol for $15 \mathrm{~min}$, followed by three washes of distilled water. Blocking solution $(1 \%(\mathrm{w} / \mathrm{v})$ BSA in TBS $(0.05 \mathrm{M}$ Tris- $\mathrm{HCl}$, $0 \cdot 138 \mathrm{M} \mathrm{NaCl}$ and $0 \cdot 0027 \mathrm{M} \mathrm{KCl}, \mathrm{pH} 8 \cdot 0)$ ) was applied for $1 \mathrm{~h}$ at $25^{\circ} \mathrm{C}$, followed by incubation with primary antibody for $16-18 \mathrm{~h}$ at $4{ }^{\circ} \mathrm{C}$ in a humidified chamber. The primary antibody solution was aspirated, and the slides were washed three times at $25^{\circ} \mathrm{C}$ in TBS. Biotinylated anti-mouse antibody (Vector Laboratories) or biotinylated anti-rabbit antibody (Jackson Immunoresearch Laboratories) was prepared according to the manufacturers' instructions and applied for $60 \mathrm{~min}$ at $25^{\circ} \mathrm{C}$, followed by three TBS washes as above. ABC-HRP was prepared according to the manufacturer's instructions, applied for $30 \mathrm{~min}$ and then washed as above. The peroxidase substrate solution was then applied to each section for $5 \mathrm{~min}$. The reaction was terminated by aspiration of the substrate solution followed by washing for $10 \mathrm{~min}$ in distilled water at $25^{\circ} \mathrm{C}$. The sections were counterstained with hematoxylin, dehydrated, coverslipped and images were digitized.

\section{Results}

IGFR-2 immunolocalization is diminished by DEX in the IECs of extremely low birth weight neonates and newborn mice

Because we were interested in perturbations of the IGF system that might have clinical correlations with neonatology, immunohistochemistry was used to examine IGFR-2 abundance in newborn mouse and human neonatal ileum and we compared specimens that were and were not exposed to early postnatal DEX by parallel processing (Fig. 2A-D). We hypothesized that IECs in the 


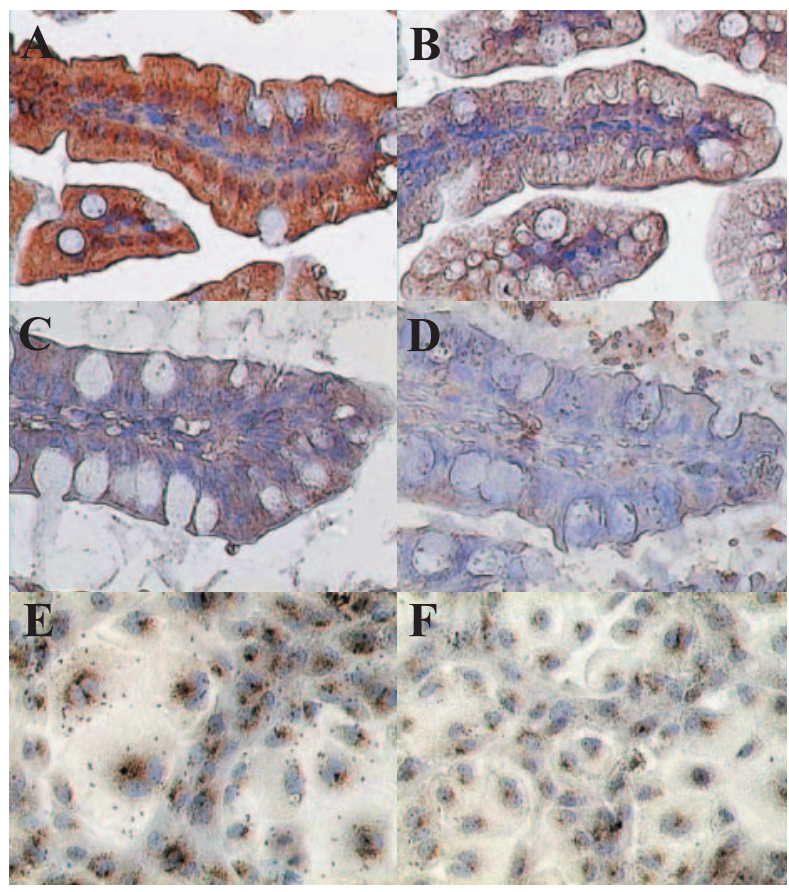

Figure 2 Representative IGFR-2 immunolocalization in (A and B) newborn mouse ( $n=8$ each), $(C$ and $D)$ human neonatal ileum $(n=3$ each) that either (B and D) were or (A and C) were not exposed to at least $72 \mathrm{~h}$ of early postnatal DEX administration (magnification $=40 \times)$. Also, $(E$ and $F)$ rat IEC-18 cells were grown to confluence in serum free media (SFM) and then switched to either (E) new SFM or (F) SFM $+10^{-6} \mathrm{M}$ DEX for $72 \mathrm{~h}$ before being fixed and processed for IGFR-2 immunolocalization. All samples were processed and detected in parallel for semi-quantitative comparison of IGFR-2 staining abundance between treatment conditions. In each case, DEX treatment was associated with reduced IGFR-2 abundance within IECs when compared with controls.

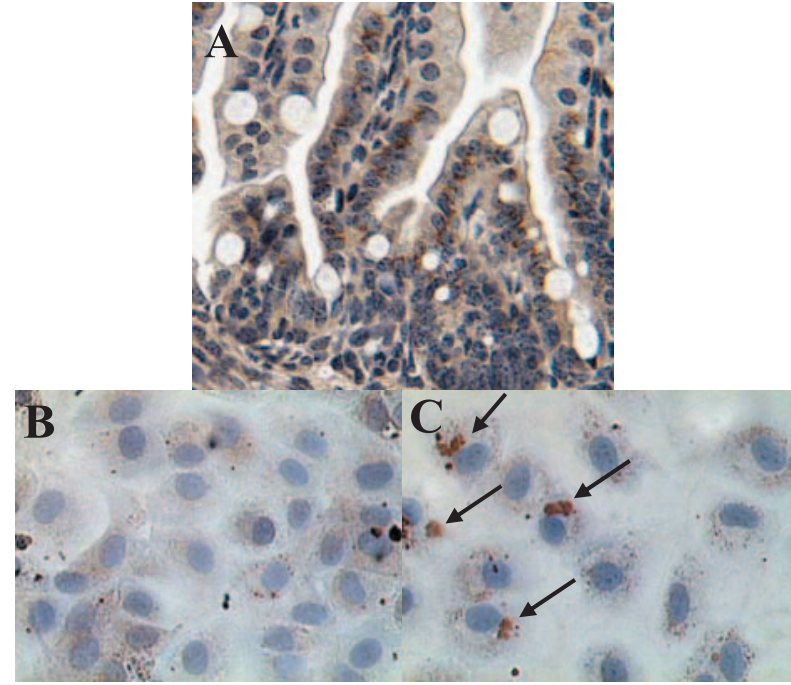

Figure $3 \mathrm{Immunolocalization}$ of IGF-II in (A) newborn mouse ileum and $(B$ and $C$ ) rat IEC-18 cells (magnification $=40 \times$ ). IGF-II is localized within the proximal IECs of the villus in a punctate, perinuclear pattern indicating its availability to proliferating IECs. IEC-18 cells were grown to confluence in SFM then switched to either (B) new SFM or (C) SFM+1.5 $\times 10^{-6} \mathrm{M} \mathrm{NBI} 31772$ (an agent that liberates IGFs from IGFBPs) for $72 \mathrm{~h}$ before being fixed and processed for IGF-II in parallel. IGF-II is sequestered in NBI 31772-treated cells - demonstrating that IGF-II is prevalent and actively endocytosed. In contrast, IGF-I was not found to be sequestered in NBI 31772-treated cells by immunohistochemistry, but was evident in positive histologic controls (newborn mouse ileum - data not shown). The arrows point to cells with positive punctate staining for IGF-II.

fetal-to-perinatal window of gut development would exhibit steroid-responsive decreases in IGFR-2 abundance. In newborn mice (representative images shown in

Table 1 Gene array subset analysis of the effect of DEX on mRNA abundance for all shown genes derived from mRNA isolated from parallel treated IEC-18 cultures (shown as means of arbitrary units of luminescence from triplicate experiments with all $P$ values $\leq 0 \cdot 05$ reported and being indicative of significant treatment effect). Note that values $\leq 10$ units indicate that detection for that probe was comparable with background levels for blanks

\begin{tabular}{|c|c|c|c|}
\hline SFM (s.D.) & DEX (s.D.) & $P$ value & Fold \\
\hline $306 \cdot 2(34 \cdot 4)$ & $1096 \cdot 9(139 \cdot 2)$ & $0 \cdot 01$ & $+3 \cdot 58$ \\
\hline $411.9(48.4)$ & $985 \cdot 3(45 \cdot 6)$ & $0 \cdot 00$ & $+2 \cdot 39$ \\
\hline $211 \cdot 2(37 \cdot 2)$ & $1312 \cdot 5(253 \cdot 1)$ & $0 \cdot 02$ & $+6 \cdot 21$ \\
\hline $5 \cdot 1(1 \cdot 8)$ & $5 \cdot 8(2 \cdot 9)$ & & \\
\hline $159 \cdot 4(8 \cdot 2)$ & $156 \cdot 6(18 \cdot 3)$ & & \\
\hline $167 \cdot 6(22 \cdot 6)$ & $230 \cdot 0(33 \cdot 0)$ & & \\
\hline $1034.5(83 \cdot 0)$ & $968.9(52.7)$ & & \\
\hline $25.9(10 \cdot 0)$ & $23 \cdot 7(13 \cdot 7)$ & & \\
\hline
\end{tabular}




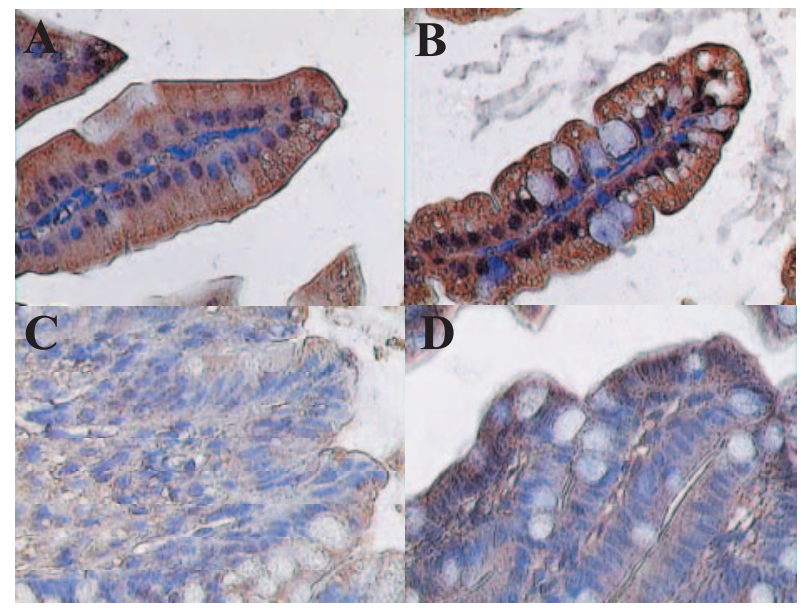

Figure 4 CREG immunolocalization in ( $\mathrm{A}$ and $\mathrm{B}$ ) newborn mouse, $(C$ and $D)$ human neonatal ileum that either $(B$ and $D)$ were or $(A$ and $\mathrm{C}$ ) were not exposed to at least $72 \mathrm{~h}$ of early postnatal DEX administration $($ magnification $=40 \times$ ). All samples were processed and detected in parallel for semi-quantitative comparison of CREG staining abundance between treatment conditions. In every case, DEX treatment was associated with increased CREG abundance within IECs when compared with those of controls.

Fig. 2A and B), IECs are larger and allow for better visualization of intracellular localization. In this animal model, IGFR-2 was associated with a more discrete perinuclear localization pattern and this staining pattern was dramatically diminished with DEX treatment. The uniformity of staining intensity between controls and DEX-treated animals was extremely consistent. In human neonatal ileum (representative images shown in Fig. 2C and D), IGFR-2 was highly abundant and most heavily localized in a perinuclear distribution within the IECs of controls but was globally diminished in DEX-exposed ileum. The uniformity of staining intensity between the controls and DEX-treated human tissues was fair but not completely homogeneous, as might be expected since they were not paired cohorts and infants acquired perforations at different times in their steroid taper.

\section{DEX decreased IGFR-2 protein but not $m R N A$ abundance in IEC-18 cells}

We utilized IEC-18 culture to further investigate the effect of DEX on IGFR-2 abundance in IECs (Fig. 2E and F). We found that IGFR-2 protein was diminished by DEX treatment in IEC-18 cells by immunocytochemistry and Western blot analysis when compared with controls, but that IGFR-2 mRNA was unchanged by DEX when investigated by gene array analysis (Table 1). We noted that all cells showed immunopositivity for IGFR-2 in a single punctate compartment, consistent with its role as a lysosomal trafficking receptor.

IGF-II is the predominant ligand driving IEC proliferation in vivo and in culture

To test the postulate that IGF-II is the predominant IGF ligand utilized for IEC proliferation, three experiments were performed. First, immunohistochemistry for IGF-I and IGF-II was performed in the proximal aspects of

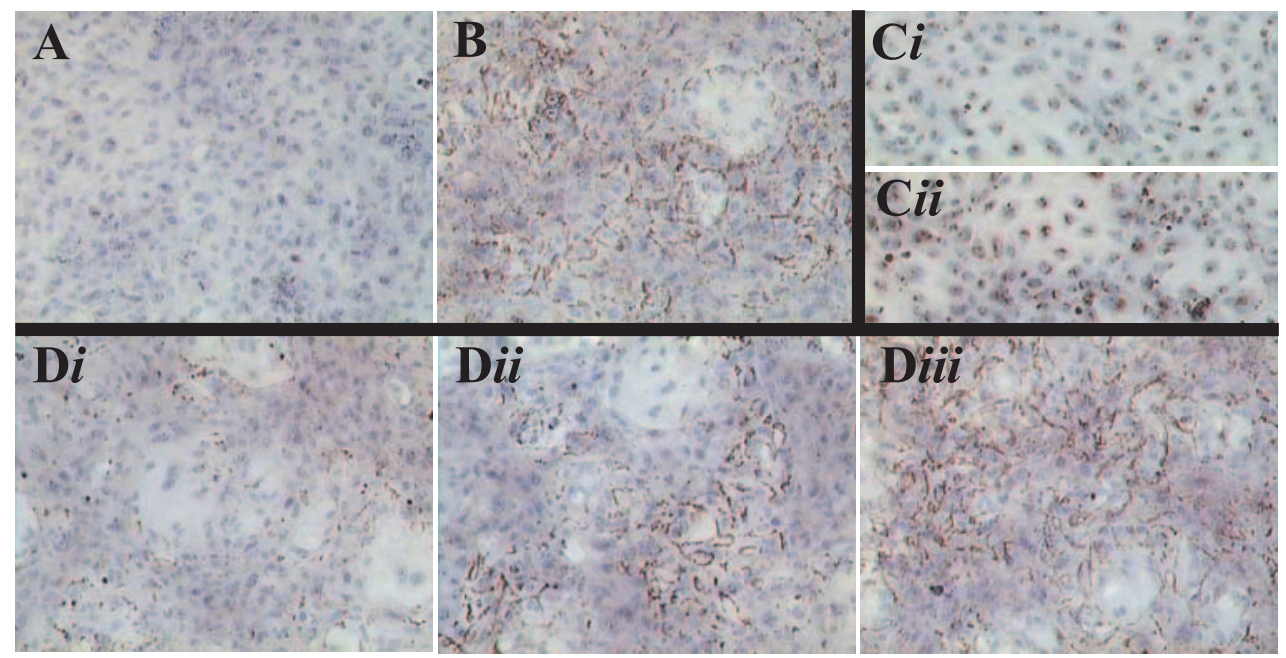

Figure 5 (A, B and Di-iii) CREG and (Ci and Cii) IGFR-2 immunolocalization in confluent IEC-18 cells treated for $72 \mathrm{~h}$ in either (A) SFM or (B and Ci) DEX. In addition, DEX treatment was performed in concert with immunoneutralization of CREG with anti-CREG polyclonal antisera at concentrations of (Cii and Di) 1:80, (Dii) 1:160 and (Diii) 1:320. Immunoneutralization of CREG (Di-Diii) reduced CREG immunostaining in a concentration-dependent manner and also (Ci versus Cii) prevented DEX-induced loss of IGFR-2 immunolocalization. 
ileal villi of newborn mice to look for sequestration of ligand. IGF-II was localized in crypt and neck cells in a punctate perinuclear pattern (Fig. 3A) whereas IGF-I was not visualized above background staining in these cells (although it was present in the submucosa - not shown), consistent with IGF-II sequestration in proliferating and newly divided cells. Secondly, IEC-18 cells were cultured for $72 \mathrm{~h}$ in SFM either with or without NBI 31772 (a chemical that displaces IGFs from all IGFBPs, resulting in increased IGF availability to the IGF receptor and subsequent endocytosis) and then processed in parallel for either IGF-I or IGF-II immunocytochemistry following NBI 31772 treatment. IGF-II was found to be localized in a punctate vesicular pattern consistent with sequestration into the cell following NBI 31772 treatment (Fig. 3B and C) whereas IGF-I was not seen above background staining - not shown. Finally, gene array data was used to look at the mRNA abundance of IGF-I and IGF-II in IECs. While comparisons across probes are not strictly quantitative because of differences in hybridization optima, IGF-II was detected in moderate abundance and did not appear to change with DEX treatment whereas IGF-I was not detected above the background level of a null hybridization (Table 1).

CREG immunostaining is increased by DEX in IECs of extremely low birth weight neonates and newborn mice

To determine if DEX alters CREG abundance in vivo, archived ileum from newborn mice and extremely low birth weight infants that were or were not exposed to DEX during the first days of life were utilized for CREG immunohistochemistry. The ileums that were exposed to DEX had increased staining within the IECs when compared with controls that were processed in parallel (Fig. 4). As with IGFR-2 immunostaining, uniformity among the neonatal mice was extremely homogeneous whereas the staining was generally less robust in the human specimens and there was greater variation between DEX-exposed patients, suggestive of the importance of timing of steroid exposure. These findings indicated that DEX increases CREG accumulation within IECs in vivo, beyond the levels of that stimulated by normal cortisol levels. We also noted that there was no detected CREG staining in other cell types such as the fibroblasts, lymphocytes or macrophages, which are found with the lamina propria, and this was unchanged with DEX treatment, further suggesting that CREG expression is specific to IECs.

\section{DEX treatment induces CREG glycocalyx deposition in IEC-18 culture}

To determine if DEX can induce CREG synthesis and secretion, IEC-18 cells were treated with either DEX or nothing for 3 days and then harvested for comparative immunochemical studies (Fig. 5). Immunolocalization of
CREG resulted in a peculiar set of observations. CREG was minimally detected in untreated cells but staining was present in two planes of focus within DEX-treated culture (not portrayed in the figures which are at lower magnification). There was a low level of staining within the cytoplasm of cells, consistent with de novo synthesis and similar to the staining seen in IECs in vivo, but with DEX treatment there was also a very intense immunoprecipitate that required focus immediately above the plane of the IECs (Fig. 5B). This staining pattern was reminiscent of 'browned meringue' and is consistent with deposition of CREG within the glycocalyx that sits on top of the cell confluency. Accordingly, dot blot comparisons showed detection of CREG in DEX-treated cell lysates but not in DEX-treated media, with minimal detection in the control lysates (Fig. 6A). Gel densitometry of multiple experiments confirmed that the DEX-induced increase in CREG abundance meets the definition of statistical significance (Fig. 6B). This same antibody was unable to detect CREG by Western blot at the same sensitivity (picomolar - suggesting a loss of native antigen during the process of Western blotting) but, at the femtomolar level of sensitivity, three bands were detected in DEX-treated lysates whereas only two were detected in SFM (Fig. 6C). The third band was a $28 \mathrm{kDa}$ band, appropriate in size for glycosylated CREG, whereas the other two bands were the predicted size for a recently identified glycosylated and non-glycosylated CREG family member known as CREG2 (Kunita et al. 2002). These putative CREG2 bands were also increased in quantity by DEX treatment.

\section{IGFBP-3 is an appropriate comparison protein for DEX experiments in IEC-18 cells}

IGFBP-3 was chosen as the control protein for cell lysate comparisons in DEX-treated conditions because of the authors' prior experience with this protein in this cell line. IEC-18 cells do not accumulate IGFBP-3 into the media (presumably because they do not make the acid labile subunit) but have reliably detectable IGFBP-3 which immunolocalizes to the cell surface and is not altered in abundance by $72 \mathrm{~h}$ of DEX treatment (authors' unpublished data). DEX also does not alter IGFBP-3 mRNA abundance (Table 1).

\section{Immunoneutralization of CREG attenuates DEX-induced degradation of IGFR-2}

To determine if CREG mediates DEX-induced degradation of IGFR-2, immunoneutralization experiments were performed in IEC-18 culture. Western blots of IGFR-2 in DEX-treated cell lysates that were coincubated with 1:80 of anti-CREG polyclonal antibody showed increased IGFR-2 when compared with DEX-treated cell lysates alone (Fig. 6D). This effect was lost in a concentration-dependent manner as the antibody 

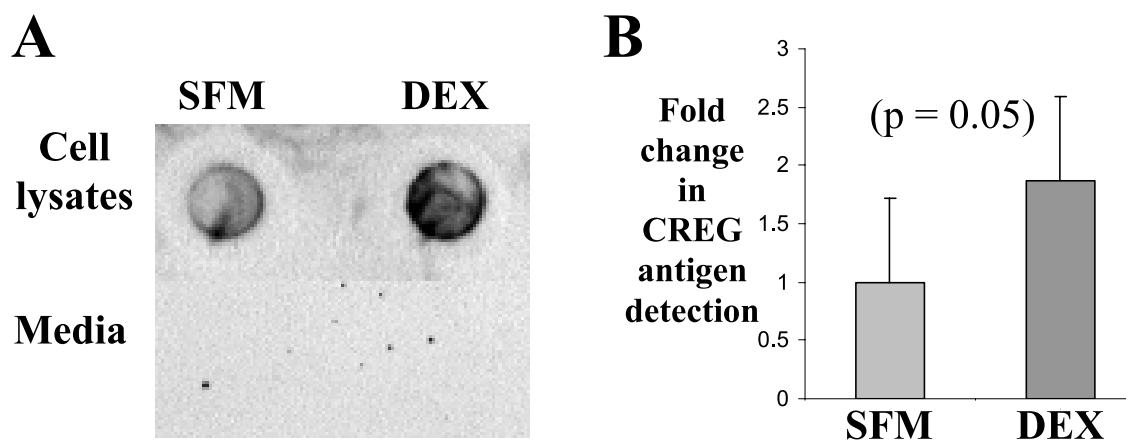

C

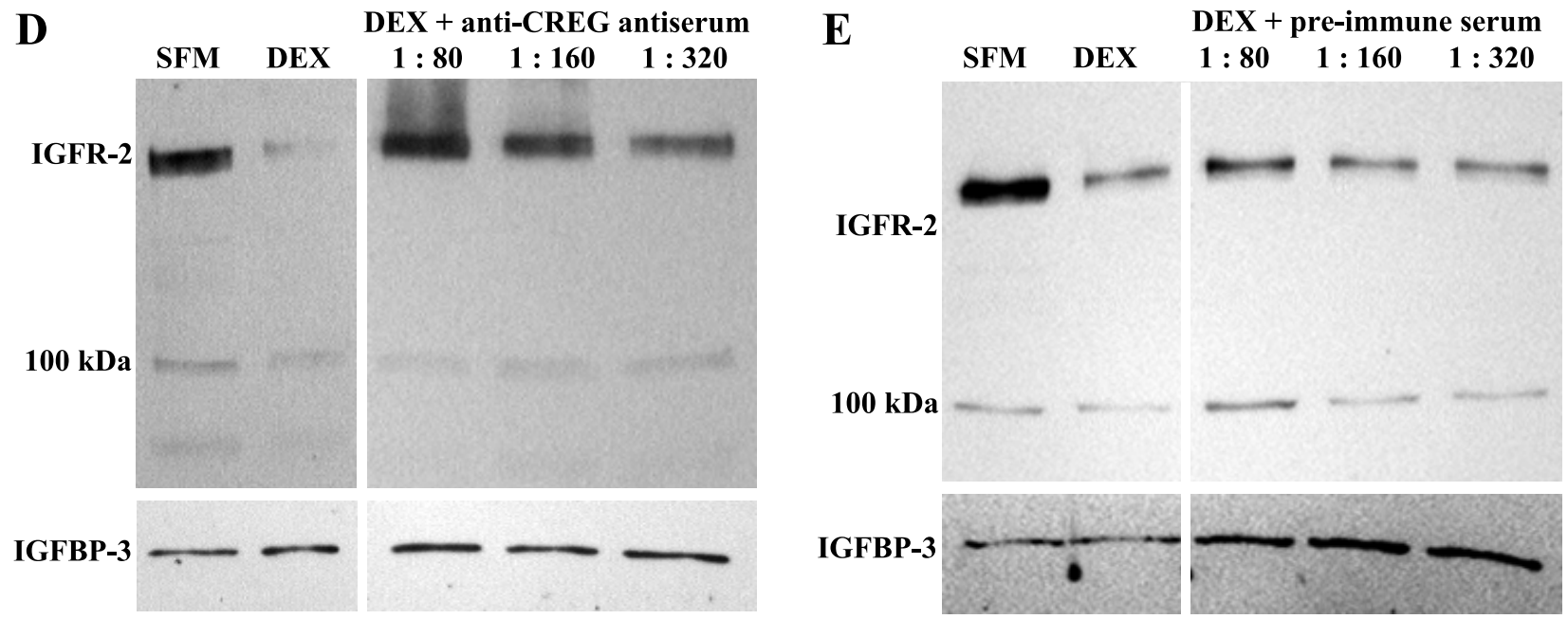

Figure 6 (A) Dot blots of CREG antigen in cell lysates and media from cells grown in SFM with and without DEX for $72 \mathrm{~h}$. CREG antigen is more abundant in DEX-treated cell lysates than in SFM. CREG antigen is not detected in media. (B) Gel densitometry of CREG antigen dot blots from three experiments demonstrating a significant increase in CREG within cell lysates of DEX-treated IEC-18 cells $(P=0 \cdot 05$ by a one-tailed t-test). (C) Western blot of CREG in cell lysates using femtomolar sensitivity for detection. Bands at 32 and $34 \mathrm{kDa}$ are seen in both SFM- and DEX-treated conditions, although they are increased in quantity in DEX treatment. These are the predicted band sizes for glycosylated and non-glycosylated forms of the brain-derived CREG family member known as CREG2. In contrast, a 28 kDa band (the correct size for glycosylated CREG) is abundant in DEX-treated lysates but is minimally detected in SFM conditions. (D and E) Immunoneutralization experiments in which confluent IEC-18 cells were incubated either in SFM or SFM+DEX with variable dilutions of (D) CREG antiserum or (E) non-immune antiserum for $72 \mathrm{~h}$ before being harvested for cell lysates. The resulting Western blots demonstrate changing abundance of IGFR-2 with the same membranes stripped and re-probed for IGFBP-3 for protein loading comparison. In IEC-18 cells, IGFBP-3 is found as a single $35 \mathrm{kDa}$ band in cell lysates that immunolocalizes to the cell surface and is not detected in the media (authors' unpublished results). Its abundance does not change with DEX exposure (D and E). However, IGFBP-3 is increased slightly in all serum-treated conditions, consistent with induced protein synthesis and a slight increase in gel protein load (D and E). DEX diminishes IGFR-2 but immunoneutralization with anti-CREG antisera prevents this in a concentration-dependent manner (D), whereas non-immune serum does not (E). Note that IGFR-2 in DEX-treated conditions does not display a prominent peptide degradation ladder as might be expected with a polyclonal antibody-derived Western blot documenting an increase in cell surface proteolysis (the homeostatic mechanism of IGFR-2 degradation) but rather is diminished without the addition of new peptide fragments or an increase in the base-line abundance of the normal degradation fragment seen at $100 \mathrm{kDa}$. This negative finding is more consistent with either lysosomal or peroxisomal degradation, neither of which generate peptide maps on polyclonal Western blots.

was diluted to $1: 160$ and 1:320. Immunocytochemistry of CREG done in parallel at low magnification revealed that CREG staining intensity in the glycocalyx was inversely proportional to the amount of antibody in the culture media, while diffuse staining within the cell cytoplasm (consistent with nascent CREG) was unaffected (Fig. 5E). While not quantitative, this demonstrated a homogeneous effect that parallels that of antibody dilution. We note that this was the only measure of CREG that we were able to perform to verify a change in abundance (because dot blots are affected by neutralizing antibody in the media and our antibody displays reduced antigen affinity in Western blots). Finally, parallel experiments with non-immune serum did not alter the effect of DEX upon CREG or upon IGFR-2, further confirming a specific antibody effect (Fig. 6E). 


\section{Discussion}

CREG is a relatively recently described glycoprotein whose function and role in development and tissue growth is still being explored (Clairmont \& Czech 1989, Veal et al. 2000, Di Bacco et al. 2003). In this paper, we have demonstrated that CREG was induced by DEX in IEC-18 cells and that CREG accumulation (assessed by immunostaining) was increased by DEX exposure in the neonatal ileum. While there may be many downstream effects from this induction, we focused on its effect upon IGFR-2 abundance. Based on our immunoneutralization studies, DEX induction of CREG diminished IGFR-2 protein without affecting IGFR-2 mRNA, consistent with an increase in IGFR-2 protein degradation.

Many of the effects of CREG could potentially be explained by degradation of IGFR-2. The function of IGFR-2 in the fetus is to prevent IGF-II-driven overgrowth of tissue by binding and internalization of free IGF-II, and then carriage of IGF-II to the pre-lysosome for eventual lysosomal destruction. In essence, this forces the IGF receptor to receive IGF-II from IGFBPs - which are tightly regulated and therefore not permissive for tissue overgrowth. Likewise, during periods of cell fate transition, excess IGF is often required to proceed. Thus an excess of IGFR-2 could be the gate that prevents that progression. Rapid degradation of IGFR-2 could therefore facilitate tissue hypertrophy in some tissues or permit maturation in others, depending on the window of development. The most logical mechanism by which CREG could mediate this is through ligand-driven degradation. In such a model (Fig. 1), the IGFR-2-CREG complex fails to disassemble in the presence of a lowered $\mathrm{pH}$, traffics to the lysosome and is degraded by acid hydrolases.

IGFR-2 plays an intrinsic role within the proximal-todistal growth disparity along the fetal gut and this is becoming an increasingly important anatomical caveat to clinical neonatology. This often overlooked conundrum of growth restriction is reinforced by the presence of 11ß-hydroxysteroid dehydrogenase (an enzyme that reduces cortisol to a less biologically active form), which is expressed disproportionately in the distal intestine (Clairmont \& Czech 1991, Pacha \& Miksik 1994, Claus et al. 2001). This enzyme limits glucocorticoid-induced maturation of tissue during neonatal development but the enzyme can be overwhelmed by exogenous administration of steroids which results in mucosal hypertrophy in preterm neonates and developmentally appropriate animal models (Shulman et al. 1998, Schaeffer et al. 2000, Gordon et al. 2001d, Dilsiz et al. 2003, Pacha et al. 2003). In older neonates this effect has actually been found to be protective for neonatal gut disease, presumably because of improved barrier function against colonizing pathogens (Shulman et al. 1998). However, in extremely low birth weight infants, synthetic glucocorticoids result in spontaneous intestinal perforations because of the limited capacity of the bowel wall to accommodate mucosal hypertrophy (Garland et al. 1999, Gordon et al. 2001b, Stark et al. 2001). For these reasons, it is crucial that we understand how steroids perturb intestinal development, and additional research into these mechanisms is needed to assist in guiding the management of this vulnerable patient population (Gordon 2004, Jobe 2004).

We acknowledge that there are limitations to this study. First, we acknowledge that there might be other mediators of the DEX effect working in concert or downstream of CREG (in fact CREG2 seems a likely co-mediator), some of these might not be evident in an immunoneutralization study - however, we know that CREG is a ligand for IGFR-2 (Di Bacco \& Gill 2003). Secondly, we have not actually proven that a loss of IGFR-2 is responsible for IEC proliferation in vivo, only that IEC-18 cells are IGF-II dependent in culture (by virtue of the absence of IGF-I) and that the abundance of IGFR-2 waxes and wanes appropriately in vivo. However, the literature supports the supposition that IEC proliferation is IGF-II dependent in vivo (Tremblay et al. 2001). Thirdly, while our paper puts forth several novel postulates regarding the role of CREG in IEC fate regulation, we have not manipulated CREG in vivo and therefore can only speculate about its function during fetal and neonatal development.

In summary, we have suggested a mechanism by which steroids induce proliferative hypertrophy in the ileal mucosa and have provided the first evidence that CREG mediates this effect via degradation of IGFR-2. Further work is needed to fully understand the role of the CREG family in IEC proliferation.

\section{Acknowledgements}

We thank Joshua Attridge for pertinent discussions and manuscript review. We thank Alyson Prorock for her technical expertise in performing the gene array experiments. This work was supported by NIH NIDDK grant 1KO8DK/HD61553-01 (P G). The authors declare that there is no conflict of interest that would prejudice the impartiality of this scientific work.

\section{References}

Clairmont KB \& Czech MP 1989 Chicken and Xenopus mannose 6-phosphate receptors fail to bind insulin-like growth factor II. Journal of Biological Chemistry 264 16390-16392.

Clairmont KB \& Czech MP 1991 Extracellular release as the major degradative pathway of the insulin-like growth factor II/mannose 6-phosphate receptor. Journal of Biological Chemistry 266 12131-12134.

Claus R, Raab S \& Lacorn M 2001 Activities of 11 beta-hydroxysteroid dehydrogenase 2 in different regions of the intestinal tract of pigs. Experimental and Clinical Endocrinology and Diabetes 109 374-377.

Dahms NM, Brzycki-Wessell MA, Ramanujam KS \& Seetharam B 1993 Characterization of mannose 6-phosphate receptors (MPRs) from opossum liver: opossum cation-independent MPR binds insulin-like growth factor-II. Endocrinology 133 440-446. 
D'Ercole AJ 1999 Mechanisms of in utero overgrowth. Acta Paediatrica 88 (Suppl) 31-36.

Di Bacco A \& Gill G 2003 The secreted glycoprotein CREG inhibits cell growth dependent on the mannose-6-phosphate/insulin-like growth factor II receptor. Oncogene 22 5436-5445.

Dilsiz A, Ciftci I, Aktan TM, Gurbilek M \& Karagozoglu E 2003 Are localized intestinal perforations distinct from necrotizing enterocolitis? Journal of Pediatric Surgery 38 763-767.

Garland JS, Alex CP, Pauly TH, Whitehead VL, Brand J, Winston JF, Samuels DP \& McAuliffe TL 1999 A three-day course of dexamethasone therapy to prevent chronic lung disease in ventilated neonates: a randomized trial. Pediatrics 104 91-99.

Gordon PV 2004 Postnatal dexamethasone for lung disease of prematurity. New England Journal of Medicine 350 2715-2718.

Gordon P, Rutledge J, Sawin R, Thomas S \& Woodrum D 1999 Early postnatal dexamethasone increases the risk of focal small bowel perforation in extremely low birth weight infants. Journal of Perinatology 19 573-577.

Gordon PV, Price WA \& Stiles AD 2001a Dexamethasone administration to newborn mice alters mucosal and muscular morphology in the ileum and modulates IGF-I localization. Pediatric Research 49 93-100.

Gordon PV, Young ML \& Marshall DD $2001 b$ Focal small bowel perforation: an adverse effect of early postnatal dexamethasone therapy in extremely low birth weight infants. Journal of Perinatology 21 156-160.

Gordon PV, Price WA, Stiles AD \& Rutledge JC 2001c Early postnatal dexamethasone diminishes transforming growth factor alpha localization within the ileal muscularis propria of newborn mice and extremely low-birth-weight infants. Pediatric and Developmental Pathology 4 532-537.

Gordon PV, Marshall DD, Stiles AD \& Price WA 2001d The clinical, morphologic, and molecular changes in the ileum associated with early postnatal dexamethasone administration: from the baby's bowel to the researcher's bench. Molecular Genetics and Metabolism 72 91-103.

Gordon PV, Moats-Staats BM, Stiles AD \& Price WA 2002 Dexamethasone changes the compositon of insulin-like growth factor-binding proteins in the newborn mouse ileum. Journal of Pediatric Gastroenterology and Nutrition 35 532-538.

Gordon PV, Paxton JB, Herman AC, Carlisle EM \& Fox NS 2004 Igf-I accelerates ileal epithelial cell migration in culture and newborn mice and may be a mediator of steroid-induced maturation. Pediatric Research 55 34-41.

Henning SJ \& Sims JM 1979 Delineation of the glucocorticoid-sensitive period of intestinal development in the rat. Endocrinology 104 1158-1163.

Herman AC, Carlisle EM, Paxton JB \& Gordon PV 2004 Insulin-like growth factor-I governs submucosal growth and thickness in the newborn mouse ileum. Pediatric Research 55 34-41.

Jobe AH 2004 Postnatal corticosteroids for preterm infants - do what we say, not what we do. New England Journal of Medicine 350 1349-1351.

Killian JK, Byrd JC, Jirtle JV, Munday BL, Stoskopf MK, MacDonald RG \& Jirtle RL 2000 M6P/IGF2R imprinting evolution in mammals. Molecular Cell 5 707-716.

Killian JK, Nolan CM, Stewart N, Munday BL, Andersen NA, Nicol S \& Jirtle RL 2001a Monotreme IGF2 expression and ancestral origin of genomic imprinting. Journal of Experimental Zoology 291 205-212.

Killian JK, Nolan CM, Wylie AA, Li T, Vu TH, Hoffman AR \& Jirtle RL $2001 b$ Divergent evolution in M6P/IGF2R imprinting from the Jurassic to the Quaternary. Human Molecular Genetics $\mathbf{1 0}$ 1721-1728.

Kunita R, Otomo A \& Ikeda JE 2002 Identification and characterization of novel members of the CREG family, putative secreted glycoproteins expressed specifically in brain. Genomics $\mathbf{8 0}$ 456-460.
Lau MM, Stewart CE, Liu Z, Bhatt H, Rotwein P \& Stewart CL 1994 Loss of the imprinted IGF2/cation-independent mannose 6-phosphate receptor results in fetal overgrowth and perinatal lethality. Genes and Development 8 2953-2963.

MacDonald RG, Pfeffer SR, Coussens L, Tepper MA, Brocklebank CM, Mole JE, Anderson JK, Chen E, Czech MP \& Ullrich A 1988 A single receptor binds both insulin-like growth factor II and mannose-6-phosphate. Science 239 1134-1137.

Morgan DO, Edman JC, Standring DN, Fried VA, Smith MC, Roth RA \& Rutter WJ 1987 Insulin-like growth factor II receptor as a multifunctional binding protein. Nature 329 301-307.

Oesterreicher TJ, Nanthakumar NN, Winston JH \& Henning SJ 1998 Rat trehalase: cDNA cloning and mRNA expression in adult rat tissues and during intestinal ontogeny. American Journal of Physiology - Regulatory, Integrative and Comparative Physiology 274 R1220-R1227.

Pacha J \& Miksik I 1994 Distribution of 11 beta-hydroxysteroid dehydrogenase along the rat intestine. Life Sciences 54 745-749.

Pacha J, Vagnerova R \& Bryndova J 2003 Carbenoxolone accelerates maturation of rat intestine. Pediatric Research 53 808-813.

Schaeffer C, Diab-Assef M, Plateroti M, Laurent-Huck F, Reimund JM, Kedinger M \& Foltzer-Jourdainne C 2000 Cytokine gene expression during postnatal small intestinal development: regulation by glucocorticoids. Gut 47 192-198.

Shulman RJ, Schanler RJ, Lau C, Heitkemper M, Ou CN \& Smith EO 1998 Early feeding, antenatal glucocorticoids, and human milk decrease intestinal permeability in preterm infants. Pediatric Research 44 519-523.

Solomon NS, Gartner H, Oesterreicher TJ \& Henning SJ 2001 Development of glucocorticoid-responsiveness in mouse intestine. Pediatric Research 49 782-788.

Stark AR, Carlo WA, Tyson JE, Papile LA, Wright LL, Shankaran S, Donovan EF, Oh W, Bauer CR, Saha S, Poole WK \& Stoll BJ 2001 Adverse effects of early dexamethasone in extremelylow-birth-weight infants. National Institute of Child Health and Human Development Neonatal Research Network. New England Journal of Medicine 344 95-101.

Trahair JF, Perry RA, Silver M \& Robinson PM 1987 Studies on the maturation of the small intestine in the fetal sheep. II. The effects of exogenous cortisol. Quarterly Journal of Experimental Physiology 72 $71-79$.

Tremblay E, Chailler P \& Menard D 2001 Coordinated control of fetal gastric epithelial functions by insulin-like growth factors and their binding proteins. Endocrinology 142 1795-1803.

Veal E, Eisenstein M, Tseng ZH \& Gill G 1998 A cellular repressor of E1A-stimulated genes that inhibits activation by E2F. Molecular and Cellular Biology 18 5032-5041.

Veal E, Groisman R, Eisenstein M \& Gill G 2000 The secreted glycoprotein CREG enhances differentiation of NTERA-2 human embryonal carcinoma cells. Oncogene 19 2120-2128.

Wang ZQ, Fung MR, Barlow DP \& Wagner EF 1994 Regulation of embryonic growth and lysosomal targeting by the imprinted Igf2/Mpr gene. Nature 372 464-467.

Whyte JR \& Munro S 2001 A yeast homolog of the mammalian mannose 6-phosphate receptors contributes to the sorting of vacuolar hydrolases. Current Biology 11 1074-1078.

Yandell CA, Dunbar AJ, Wheldrake JF \& Upton Z 1999 The kangaroo cation-independent mannose 6-phosphate receptor binds insulin-like growth factor II with low affinity. Journal of Biological Chemistry 274 27076-27082.

Young LE, Fernandes K, McEvoy TG, Butterwith SC, Gutierrez CG, Carolan C, Broadbent PJ, Robinson JJ, Wilmut I \& Sinclair KD 2001 Epigenetic change in IGF2R is associated with fetal overgrowth after sheep embryo culture. Nature Genetics 27 153-154.

Received 23 November 2004

Accepted 14 February 2005 